\title{
Use of a Viscous Flow Simulation Code for Static Aeroelastic Analysis of a Wing at High-Lift Conditions
}

\author{
H. Dogus Akaydin*1, Shayan Moini-Yekta*1, \\ ${ }^{1}$ Science and Technology Corporation, Moffett Field, CA 94035 \\ Jeffrey A. Housman ${ }^{* 2}$, and Nhan Nguyen ${ }^{\dagger 2}$ \\ ${ }^{2}$ NASA Ames Research Center, Moffett Field, CA 94035
}

\begin{abstract}
In this paper, we present a static aeroelastic analysis of a wind tunnel test model of a wing in high-lift configuration using a viscous flow simulation code. The model wing was tailored to deform during the tests by amounts similar to a composite airliner wing in highlift conditions. This required use of a viscous flow analysis to predict the lift coefficient of the deformed wing accurately. We thus utilized an existing static aeroelastic analysis framework that involves an inviscid flow code (Cart3d) to predict the deformed shape of the wing, then utilized a viscous flow code (Overflow) to compute the aerodynamic loads on the deformed wing. This way, we reduced the cost of flow simulations needed for this analysis while still being able to predict the aerodynamic forces with reasonable accuracy. Our results suggest that the lift of the deformed wing may be higher or lower than that of the non-deformed wing, and the washout deformation of the wing is the key factor that changes the lift of the deformed wing in two distinct ways: while it decreases the lift at low to moderate angles of attack simply by lowering local angles of attack along the span, it increases the lift at high angles of attack by alleviating separation.
\end{abstract}

\section{Introduction}

Improving fuel efficiency of airliners continues to be a major goal for commercial aircraft manufacturers. One method of increasing fuel efficiency is to decrease empty weight of the aircraft for the same payload weight and mission. Advances in composite materials continue to provide significant reductions in empty weight of the aircraft. However, structures made of composite materials are in general not only less heavy but also less stiff than their alloy counterparts. As a result, modern airliner wings tend to flex more than their predecessors that carry similar weights. For example, Boeing 787 and 777 wings have nearly the same span $(\approx 200 \mathrm{ft})$; however, it is reported that nominal tip deflection of the all-composite wing of a Boeing 787 at cruise is $10 \%$ of its semi-span ${ }^{1}$ whereas that of an all-alloy Boeing 777 wing is estimated to be $6.4 \%$ of its semi-span. ${ }^{2}$ Since aerodynamic loads change during different flight stages (decrease in total weight as fuel is expended, non-1g maneuvers such as take-off and landing, etc.), a capability to predict resultant shape of such flexible wings under different flight conditions has become crucial to aircraft design.

Variability of aerodynamic loads during flight has also led to efforts in dynamically adapting the wing shape to flight conditions. An example is the Variable Camber Continuous Trailing Edge Flap (VCCTEF) concept that Boeing is developing in collaboration with NASA. ${ }^{3}$ The VCCTEF concept replaces the conventional outboard flap and aileron with multiple short-span flaps that are blended to each other with a flexible elastomer material. Moreover, each VCCTEF consists of multiple chordwise elements that allow

\footnotetext{
*Research Scientist, Applied Modeling and Simulation Branch, NAS Division, MS N258-2

${ }^{\dagger}$ Technical Group Lead, Advanced Control and Evolvable Systems Group, Intelligent System Division and AIAA Associate Fellow
} 
the individual flaps to change trailing edge camber. As such, the effective wing camber can be controlled both in spanwise and chordwise directions at a resolution higher than conventional (single-component) flap and aileron designs can provide. Therefore, the VCCTEF concept offers more control over spanwise lift distribution on the wing and thus adapts better to changing flight conditions for increased fuel efficiency.

From a computational fluid dynamics point of view, on the other hand, increased structural flexibility and number of independent components make high-fidelity modeling and simulation of these aircraft more difficult. For example, the fact that the wing will change shape due to different aerodynamic loads requires us to know those aerodynamic loads, which are, in turn, determined by the wing shape. Many of the aeroelastic analysis procedures approach this problem by solving for aerodynamic forces and structural deformations in an alternating order thus converging to a reasonably accurate state of wing deformation and aerodynamic loads. ${ }^{4,5}$ An example is the framework used by Rodriguez et al., ${ }^{1}$ which utilizes Cart3D ${ }^{6}$ (an inviscid flow simulation code) for finding aerodynamic loads in conjunction with a beam element code to calculate bending and torsion of the wing under those loads. In this work, we integrated the Overflow ${ }^{7}$ (a viscous flow simulation code) into that framework in order to add a viscous flow prediction capability to it. Addition of such a high-fidelity approach is necessary, for example, to accurately predict the lift force a compositewing airliner has to produce during take-off or landing, because viscous effects such as separation become significant under those high-lift conditions. We thus implemented this method on a wing model at high-lift configuration, which was concurrently tested in a wind tunnel at the University of Washington Aeronautics Laboratory (UWAL). ${ }^{8}$ In this paper, we present our method and its results in comparison to the wind tunnel tests.

\section{Geometry, Flow Conditions and Methods}

The model studied in this work consists of a wing attached to a generic fuselage-like body halved along its symmetry plane, as shown in Figure 1. It has an inboard flap, a system of VCCTEF and a series of slats; all deployed to achieve a high-lift configuration. The narrow gaps between the separate slat sections were sealed with tape for both the wind tunnel tests and for the flow simulations. The model has a half-span of $b / 2=1.87 \mathrm{~m}$. For normalizing aerodynamic forces and moments, we used a reference area of $S=0.895 \mathrm{~m}^{2}$, which approximately is the half-wing's planform area and a reference length of $c=S /(b / 2)=0.487 m$, which approximately is the mean aerodynamic chord of the model. Flexural and torsional stiffnesses of the wing were tailored to mimic highly flexible wings during tests.

We ran the simulations at incoming flow conditions that correspond to a Reynolds number of $\operatorname{Re}_{\infty}=1.28 \times 10^{6}$ per meter and a Mach number of $M_{\infty}=0.055$, which corresponds to a freestream speed of $U_{\infty}=19 \mathrm{~m} / \mathrm{s}$. The concurrent wind tunnel tests by the UWAL group were run at about $21.4 \mathrm{~m} / \mathrm{s}$, but we confirmed that the differences this mismatch creates are less than $0.1 \%$ in lift and less than $1 \%$ in drag coefficients.

We generated a structured, body-fitted, overset grid system using Chimera Grid Tools $(\mathrm{CGT})^{9}$ which is a versatile and robust grid generation method for high-fidelity flow simulations around bodies involving complex geometric features such as the one we studied here. This grid generation tool set can be scripted to prepare, save, modify and run grid generation instructions for such a detailed grid system. In Figures 2 (a) and (b) we present an overall view of the surface grids of our grid system. Each patch in these figures represent a different overset grid zone, which is a structured grid zone that overlaps with its neighbors. As can be seen in Figures 2 (c) and (d), with the overset grid method, we can generate structured, body-fitted grid systems that can model complex geometric features with high near-wall resolutions needed for viscous flow simulations. The grid system generated for this work consists of 75 million vertices contained in 148 volume grid zones that cover a region that extends 30 body lengths away from the model. The majority of the grid nodes adjacent to the model surface has a wall spacing of about $y^{+}=0.2$ to 0.3 . We thus expect to capture viscous effects with sufficient accuracy using this grid. For solving the flow field we used Overflow, ${ }^{7}$ which is an implicit, Reynolds-Averaged Navier-Stokes (RANS) solver developed by NASA for structured overset grids. For obtaining the results presented here, we used a steady-state, second order, central difference scheme in Overflow with Spalart-Allmaras (SA) turbulence model. ${ }^{10}$

The grid scripts we developed for this work rely only on the initial geometry definition files and a series of instructions to generate the grids and to prepare input files for the flow solver. We designed these scripts such that they first make all the necessary surface grids, and then generate the volume grids based on these surface grids. This way, we generate a baseline surface grid once and subsequent updates to the surface grid propagate into the surrounding volume through the volume grids generated based on those new surface grids. 


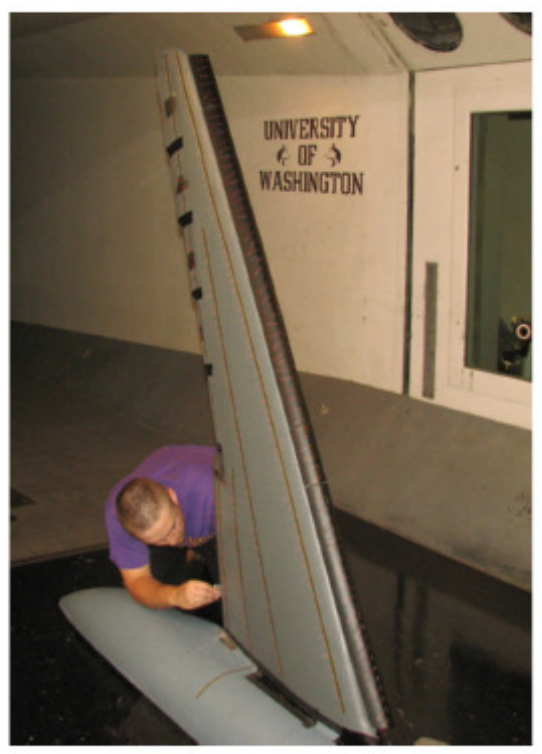

(a)

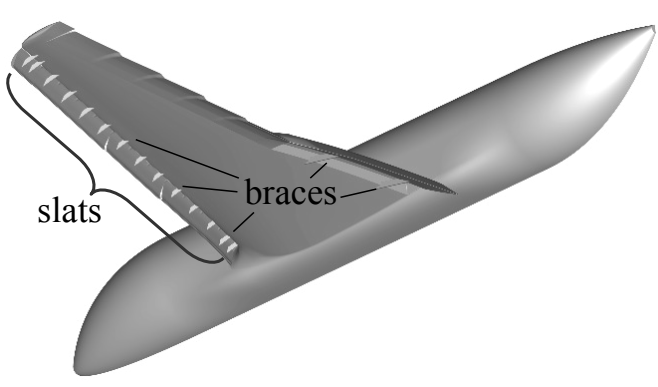

(c)

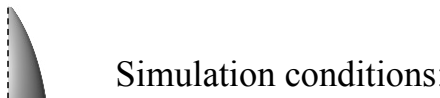

Air at $\mathrm{T}_{\text {ref }}=26^{\circ} \mathrm{C}, \mathrm{P}_{\text {ref }}=101325 \mathrm{~Pa}$

$\mathrm{U}_{\mathrm{ref}}=19 \mathrm{~m} / \mathrm{s}\left(\mathrm{M}_{\mathrm{ref}}=0.055\right)$

$\downarrow \downarrow$
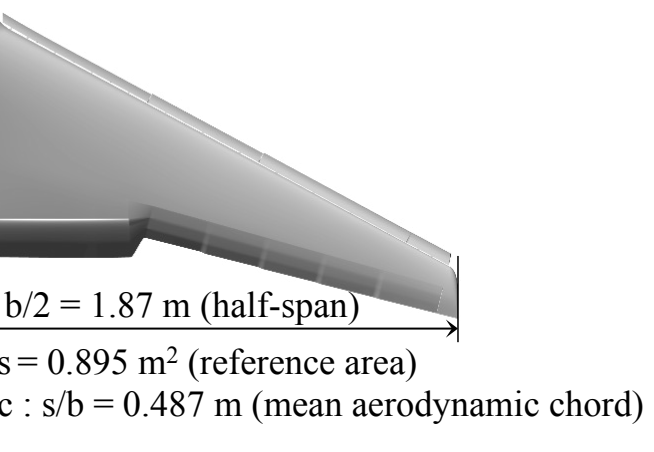

symmetry

(b)

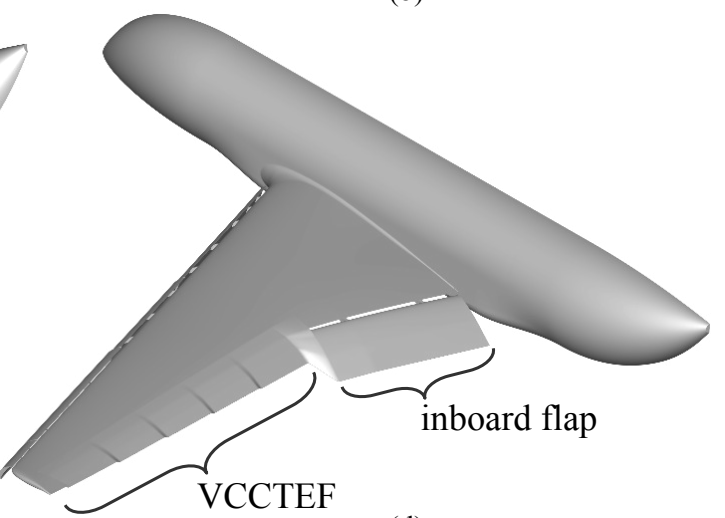

(d)

Figure 1. (a) A photograph of the wind tunnel model (courtesy of University of Washington Aeronautical Laboratory) (b) overall view of the geometry and flow conditions we used, (c) and (d) different views of the model and its components

For mild deformations without acute changes in surface grids this technique works well, and we successfully utilized it to generate the near-body grids around the deformed wings. The procedure is summarized as follows:

1. Build a structured overset grid for the original (non-deformed) wing, using the initial surface triangulations and other geometry definitions. As a reference, run Overflow simulations on this non-deformed structured grid covering a range of angle of attack from $\alpha=-8^{\circ}$ to $\alpha=+24^{\circ}$ in $4^{\circ}$ increments.

2. Repeat these sub-steps until converging to a lift coefficient and a corresponding wing shape:

(a) Run Cart3D simulations on the surface triangulations to compute the load distribution over the wing while targeting the lift coefficient obtained from the previous step. (In the beginning, use the surface triangulation and the lift coefficient of the non-deformed geometry.)

(b) Using a beam element code, bend and twist the surface triangulation according to the aerodynamic load distribution obtained in step 2(a). This will effectively displace the nodes of the surface triangulation in three degrees of freedom (translations in $\mathrm{x}, \mathrm{y}$, and $\mathrm{z}$ coordinates.).

Note that deformation in this step does preserve the span of the wing by including the foreshortening effect due to rotation of wing sections. Details of this method for deforming wings under static aeroelastic loads can be found in reference 1 . 


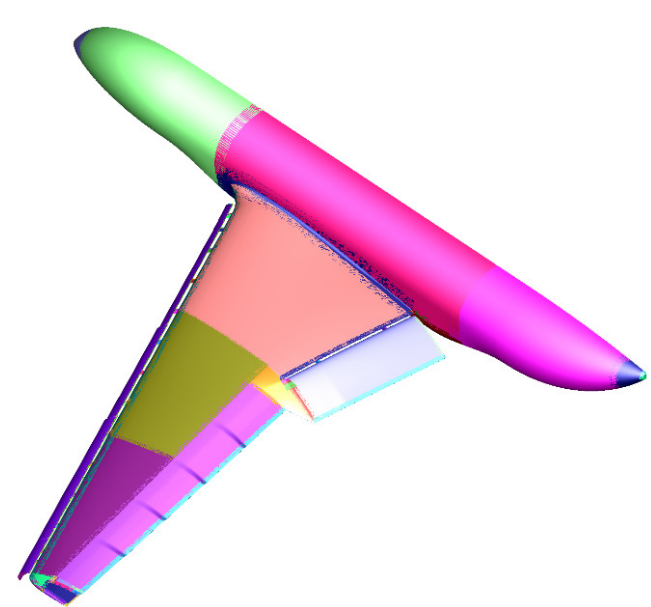

(a)

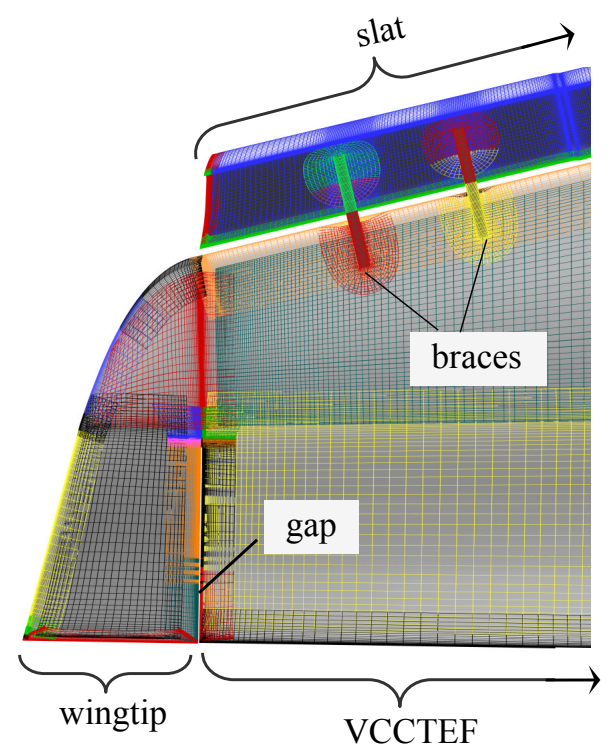

(c)

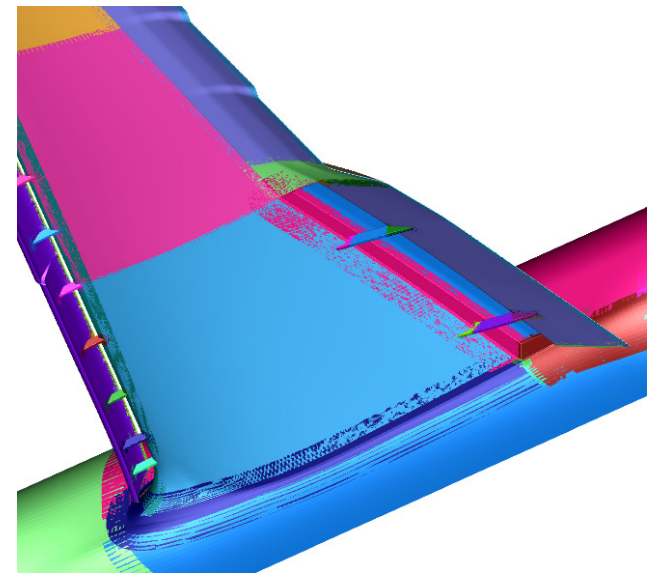

(b)

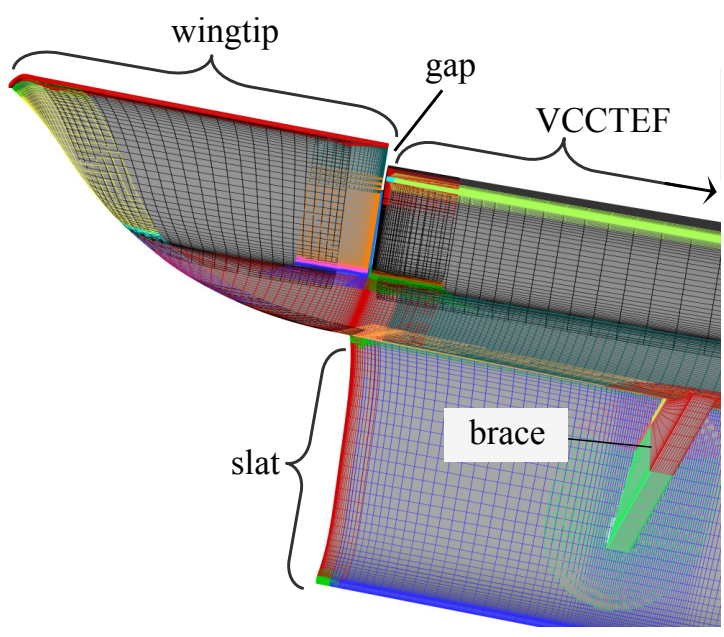

(d)

Figure 2. View of surface grid system from various angles: Overview of the grid system from (a) top and (b) bottom, looking under the wing. Each color represents a different structured overset surface grid zone. (c) and (d) Details near the wing tip showing the slat, the braces that hold the slat attached to the main element, and the narrow gap between the VCCTEF and the wing tip.

3. Generate the structured volume grid for the deformed geometry:

(a) Map the nodal displacements of the nodes of the surface triangulation to the nodes of the structured overset grid. This sub-step effectively produces a structured surface grid that is deformed in accordance with the surface triangulation.

(b) Regenerate the volume grids based on the deformed structured surface grids and perform overset grid connectivity.

Step 3 concludes the deformation of the wing. Figure 3 shows the effect of this step on two of the structured grid zones. The following steps are for finding the viscous aerodynamic loads on this deformed grid.

4. Run Overflow simulations on the deformed structured grid given by step 3, while targeting the lift coefficient predicted in step 2 and allowing the angle of attack to change. If we had fixed the angle of attack and not targeted the lift coefficient suggested in step 2, we would find a lift coefficient different than the lift coefficient for which the wing was deformed. By targeting the lift coefficient while allowing 
the angle of attack to change in this step, we ensure that the aerodynamic load on the wings is as relevant as possible to the deformed shape of the wing.

5. Run additional Overflow simulations on the non-deformed wing at select angles of attack obtained in step 4 .

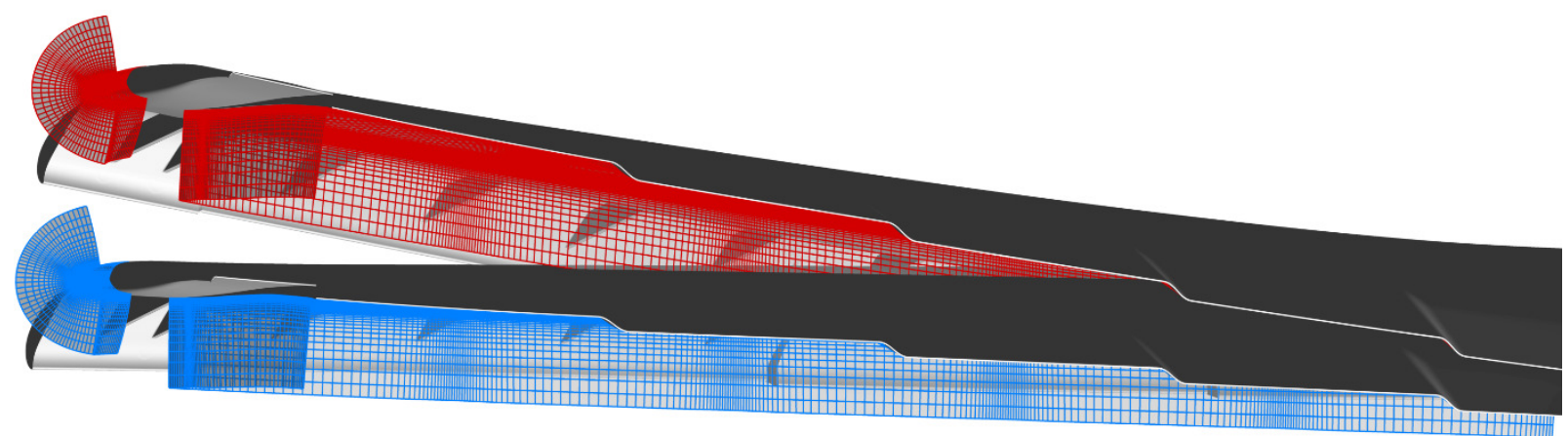

(a)

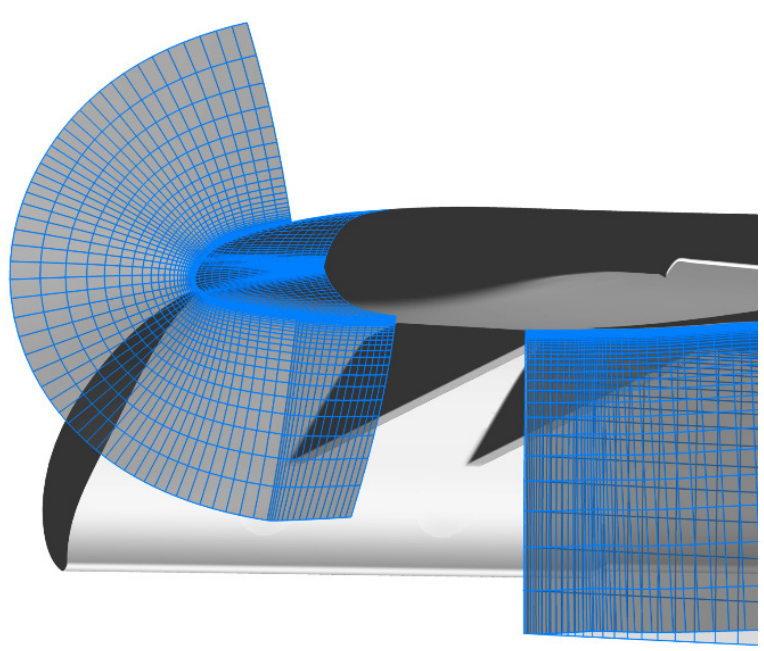

(b)

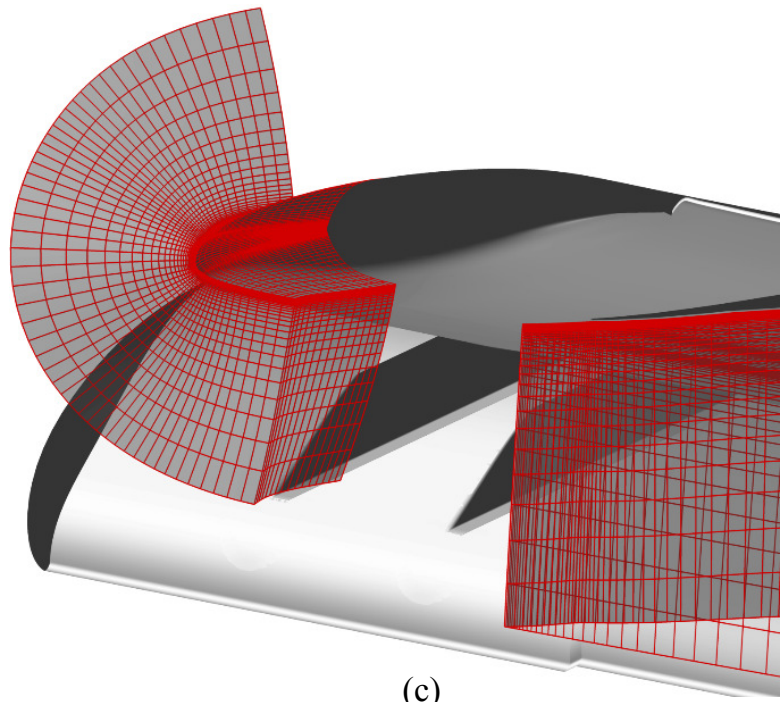

(c)

Figure 3. (a) State of two volume grids before (blue) and after (red) deformation mapping. Note that the grid that runs along the span on the bottom surface of the wing experiences a relatively large deformation whereas the grid on the wingtip experiences mostly a translation. Note also that, for clarity purposes, the grids are shown only in their half extend adjacent to the wall; they actually grow nearly twice as much into the volume out of the surfaces they are based on. (b) and (c) Details of the grids near the wingtip.

The reason we relied on an inviscid flow solver to bend the grid is twofold: First, we wanted to have a quick start by integrating a viscous analysis tool into an existing static aeroelastic framework without having to develop routines for projecting pressure distribution found on the structured surface grids to the surface triangulations. Second, even after developing those projection routines, we plan to keep using Cart3D at least in the initial iterations of Step 2, because Cart3D simulations are significantly less costly while they can capture the lift distribution reasonably well for most angles of the attack before stall. Although the Cart3D lift predictions are expected to deviate from wind tunnel tests and viscous simulations results when flow separations are prominent, as we will see in the following, they worked well enough to predict the deformed shape of the wing until $21^{\circ}$ of angle of attack on this geometry.

We ran each of these Overflow simulations in parallel on the Pleiades supercomputer at NASA Ames Research Center. Each simulation utilized 480 cores of Sandy Bridge processors and most of them converged within 50 to 100 thousand iterations, which took about 20 to 40 hours of wall time. 


\section{Results}

In Figure 4, we compare lift and drag coefficients predicted by the flow simulations on the models with deformed and non-deformed wings with those measured by the wind tunnel tests on the actual model. The simulations using the non-deformed wing geometry overpredict the lift in the regime $-4^{\circ}<\alpha \leq 16^{\circ}$, while they underpredict it beyond $16^{\circ}$ and suggests a maximum lift angle of attack much smaller than what is measured during the wind tunnel tests (about $21^{\circ}$ ). The simulations using deformed wings also overpredict the lift, however, their predictions are considerably closer to the experimental results especially at angles larger than about $4^{\circ}$. This is not surprising because at low angles of attack the wing is not heavily loaded and deformed less; as a result, the non-deformed and deformed wing shapes at low angles of attack are relatively close. As the angle of attack increases, however, the deformations on the actual wing become more pronounced; therefore, the simulations using the deformed wing predict the lift and drag coefficients better than the simulations which ignore these deformations at high angles of attack. We will now demonstrate how these deformations affect the flow characteristics, thus the total lift coefficient, by focusing on these two angles of attack: $\alpha=8^{\circ}$ and $\alpha=20.6^{\circ}$. At $\alpha=8^{\circ}$, the deformed wing produces $4.7 \%$ less lift than the non-deformed wing, and the deformations and their impact on the flow field are large enough to be visualized. This case is therefore selected as a representative of conditions when $\alpha<16^{\circ}$ On the contrary, at $\alpha=20.6^{\circ}$, the deformed wing produces $7.3 \%$ more lift than the non-deformed wing. We will therefore focus on these two converse situations to compare the structural and aerodynamic differences they have.

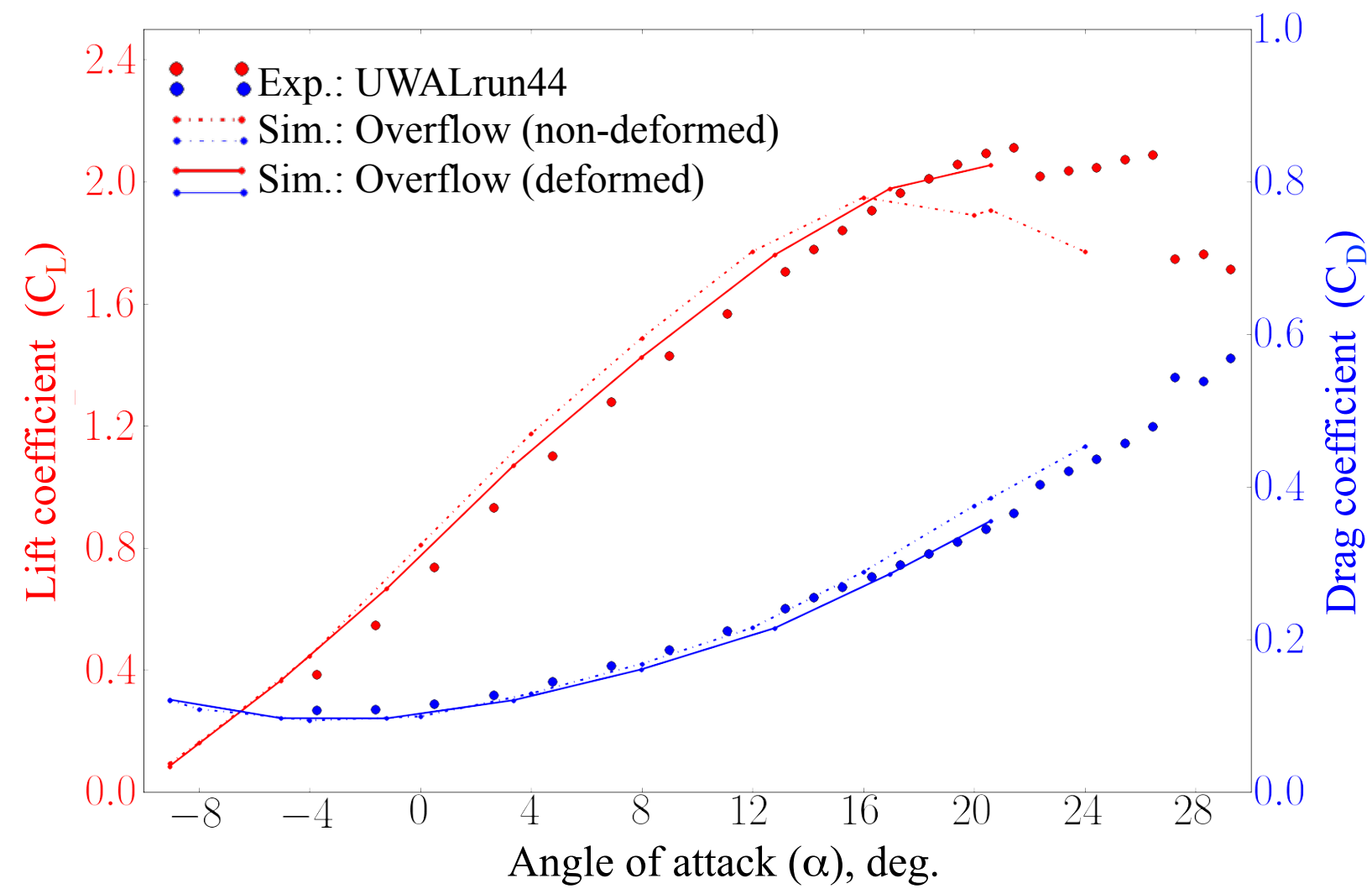

Figure 4. Lift $\left(C_{\mathrm{L}}\right)$ and drag $\left(C_{\mathrm{D}}\right)$ coefficients of the model at various angle of attack ( $\left.\alpha\right)$. "UWALrun44" refers to the data set that is obtained from the 44th run in UWAL's wind tunnel test campaign. No experimental data was taken near $\alpha=-8^{\circ}$, but we still ran simulations near that angle in order to explore the zero-lift conditions.

As a wing becomes loaded by lift forces, it bends upwards and effectively gains a positive dihedral angle. It can then be thought that this dihedral angle might be the reason for the reduction of lift; because now that the total force is turned inwards (towards the fuselage) by a certain angle, the component of it in the vertical (lift) direction should be smaller. In addition to bending, the wing may undergo twisting deformations which would affect its lift. We will now investigate which kind of deformation is actually responsible for the changes in the lift as the wing becomes loaded.

Figure 5 shows the "oil flow lines" on the top and bottom surfaces of the wings overlaid with contours of 
pressure coefficient $\left(C_{\mathrm{p}}\right)$. At $\alpha=8^{\circ}$, there is a relatively small separation region limited to the top surface of VCCTEF, and the differences between $C_{\mathrm{p}}$ contours or between the oil flow lines of the deformed and non-deformed wings are not visually striking. For this reason, we subtracted the pressure coefficient values on the non-deformed wing from those on the deformed wing $\left(\Delta C_{\mathrm{p}}=C_{\mathrm{p}}(\right.$ def. $)-C_{\mathrm{p}}$ (non-def. $\left.)\right)$ to make the changes in pressure coefficients more distinguishable. The contour plots of $\Delta C_{\mathrm{p}}$ for this case show that the deformed wing creates gradually less suction on the top surface $\left(C_{\mathrm{p}}(\right.$ def. $)>C_{\mathrm{p}}$ (non-def. $\left.)\right)$ and less pressure on the bottom surface $\left(C_{\mathrm{p}}\right.$ (def.) $<C_{\mathrm{p}}$ (non-def.) along the leading edge.

At $\alpha=20.6^{\circ}$, however, a saw-tooth shaped separation pattern, which actually stems from the braces that connect the slats to the main element, becomes the dominant feature of the flow over the wing. Furthermore, there are now striking differences between the pressure coefficient contours and the oil flow lines on the deformed and non-deformed wings: The large separation zone observed between $y=0.7$ and $y=1.1$ sections of the non-deformed wing disappears when the wing is deformed. This creates a remarkable decrease (beyond -1.5) in pressure coefficient over a large zone centered around the $y=0.9$ location near the leading edge, as seen on the corresponding $\Delta C_{\mathrm{p}}$ contours on the top surface. We can therefore state that the lift of the deformed wing at this angle is higher because there is less flow separation on the top surface of the wing as compared to the non-deformed wing. However, we do not yet know the mechanism that reduces the lift of the deformed wing at low angles of attack but increases it at some high angle of attack. Therefore we will now look at the changes that occur both in lift distribution and the wing structure at several cross sections taken in planes perpendicular to the spanwise direction.

Figure 6 shows the spanwise lift distributions in conjunction with the pressure coefficients and wing shapes at various sections. At $\alpha=-9.1^{\circ}$, which creates a minimal amount of lift, the difference in spanwise lift distribution of the deformed and non-deformed wings is relatively insignificant since the wing is not significantly loaded. At $\alpha=8.0^{\circ}$, however, the lift force creates a significant deformation (110 mm, or $5.6 \%$ of half-span) that changes the spanwise load distribution. Notably, the difference between the lift distributions at this angle is such that the deformed wing produces gradually less lift than the non-deformed one from wing root to tip. (The corresponding $\Delta C_{\mathrm{p}}$ plots in Figure 5 indeed shows a gradual decrease in pressure difference between the top and bottom surfaces.) At $\alpha=20.6^{\circ}$ (with a tip deflection of $168 \mathrm{~mm}$ or $9 \%$ of half-span), on the other hand, we observe that the lift distributions for deformed and non-deformed cases, as well as their differences, no longer change gradually from wing root to wingtip as they do in the $\alpha=8.0^{\circ}$ case. The reason for this can readily be seen in the $\Delta C_{\mathrm{p}}$ plot for $\alpha=20.6^{\circ}$ in Figure 5: on the top surface, the deformed wing produces more suction or less suction in an alternating way along the leading edge. This alternating behavior is in fact related to the saw-tooth shaped separation pattern which results in zones of high or low pressure along span near the leading edge.

At this point we can state that the dihedral effect alone does not explain the differences in lift of the deformed and non-deformed wings: First, for the $\alpha=8.0^{\circ}$ case, even if we assume that the lift is uniformly distributed along the span, a tip deflection of $110 \mathrm{~mm}$ will cause only a few degrees of rotation of the resultant sectional forces towards the fuselage, which could reduce the lift only by $1 \%$ or less. This difference is not sufficient to explain the $4.7 \%$ decrease in lift at $\alpha=8.0^{\circ}$. In addition, the rotation of the resultant force towards the fuselage can only reduce the lift; thus it certainly does not explain the increase in lift that we observe at $\alpha=20.6^{\circ}$. We thus focus our attention to the other mode of the wing deformation, namely the twisting of the wing.

It can be observed in Figure 6 that the wing cross sections not only deform but also rotate in pitch-down (washout) sense by angles $\delta(y)$, as we proceed from wing root to wing tip. This untwisting deformation along the span reduces sectional angles of attack in both cases; but it impacts the two cases very differently: While it simply reduces the lift throughout the span at $\alpha=8.0^{\circ}$; it increases the lift by alleviating separation on the top surface at $\alpha=20.6^{\circ}$. We can thus conclude that the main reason for the changes in the lift of this wing after deformation is the increase in washout of the wing due to loading, and that while this induced washout deformation may decrease the lift at a certain angle of attack, it may increase the lift at a higher angle of attack by reducing separation over the top surface of the wing.

\section{Conclusions}

In this computational work, we performed a static aeroelastic analysis of a wind tunnel test model in high-lift configuration. Our goal was to integrate a viscous flow simulation code, namely Overflow, into an existing static aeroelastic analysis framework which readily used an inviscid flow simulation code, Cart3D. 


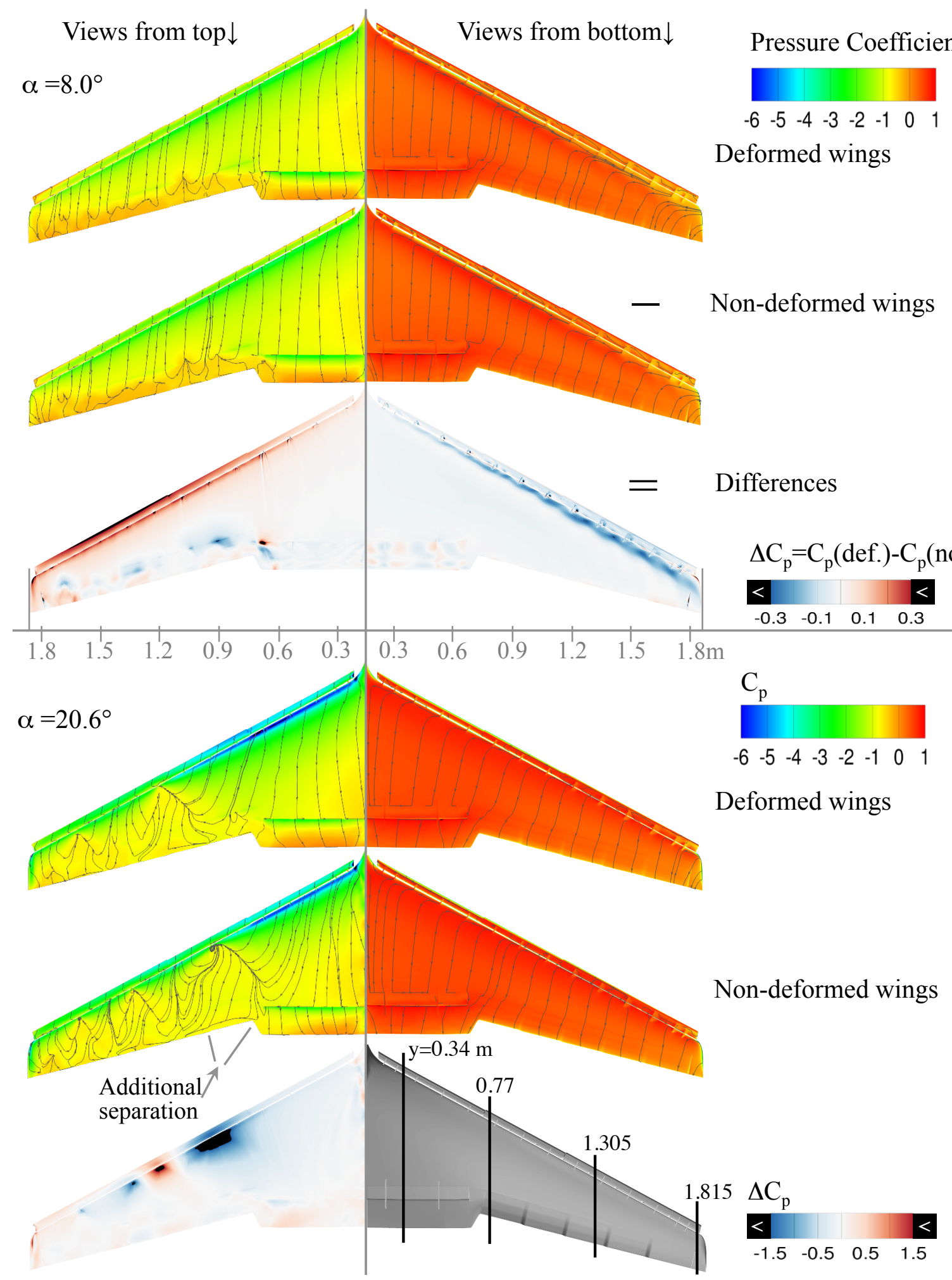

Figure 5. Contours of pressure coefficient $\left(C_{\mathrm{p}}\right)$ on top and bottom surfaces of the deformed and non-deformed wings at the two angles of attack investigated here. Also shown are the point-by-point differences $\left(\Delta C_{\mathrm{p}}\right)$ between the pressure coefficients of the deformed and non-deformed wings at both angles of attack. Note for $\alpha=20.6^{\circ}$ case that the differences between the pressure coefficients on the bottom surface due to deformation turned out to be insignificant in comparison to those on top surface, thus $\Delta C_{\mathrm{p}}$ contour for the bottom surface for that case is left out). Instead, the locations of the sections that are investigated in Figure 6 are displayed. 

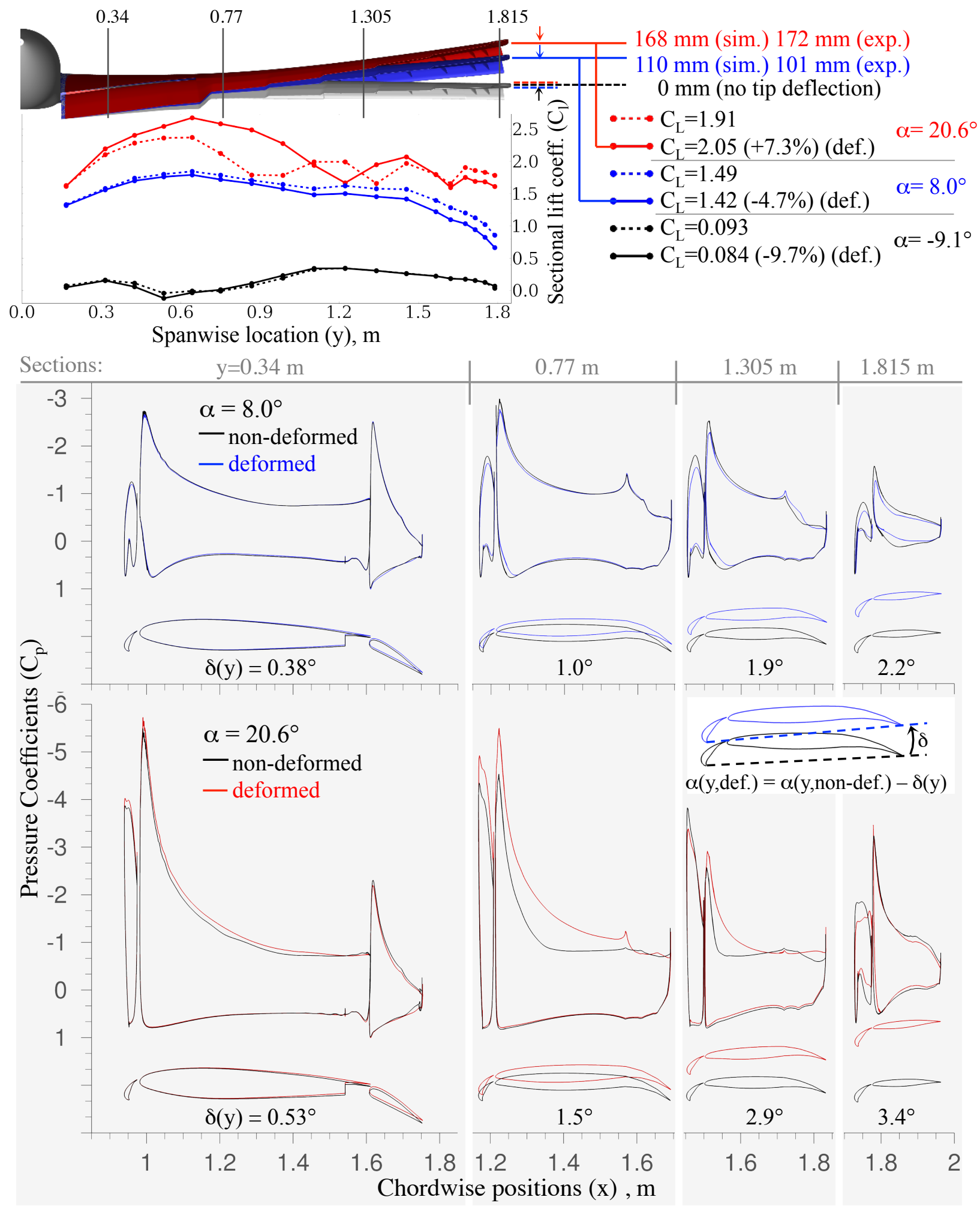

Figure 6. Spanwise distribution of $C_{1}$ for three angles of attack, in conjunction with the pressure coefficient and wing section plots at various cross sections for two of those angles of attack. Tip deflections observed at the two angles of attack in simulations (sim.) and experiments (exp.) are also indicated. 
We first found the deformed shape of the wing at a certain lift coefficient using this framework, then found a better approximation to the aerodynamic loads on that deformed geometry using Overflow. This way, we utilized relatively inexpensive methods (Cart3D and beam element codes) to converge to a deformed shape, then used a more expensive method to fine-tune the aerodynamic predictions on that deformed geometry. This method worked well in a range of angle of attack from $-8^{\circ}<\alpha<21^{\circ}$ on this configuration.

The results of this study suggest that twisting, not bending, of the wing is the main reason why the deformed wing studied here has a different lift coefficient than the non-deformed one. Although the wingtip can move upwards by $10 \%$ of the wing span, the dihedral effect would reduce the lift coefficient less than $1 \%$. Moreover, in some cases we observe an increase in the lift due to deformation, which cannot be explained by dihedral effect since it can only cause a reduction in the lift. It turns out that the major reason for these changes in the lift is twisting of the wing in pitch-down (washout) sense as it becomes deformed under lift forces. This twisting deformation reduces the angle of attack of wing sections from root to tip by up to a few degrees at the wing tip, and causes the deformed wing to have a reduced twist distribution as compared to the non-deformed wing. The interesting observation is that, while this washout deformation reduces the lift of the deformed wings below a certain angle of attack, it increases the lift after that angle by alleviating separation and delaying stall. The mechanism explained is therefore relevant to the design of composite-wing aircrafts that are expected to experience high-lift conditions.

\section{Acknowledgements}

The authors thank the following individuals for their contributions in this work: James Jensen (Graduate Intern, Arizona State University), who prepared the initial CAD definitions and generated a significant part of the initial grid system, and David L Rodriguez (Research Scientist, Science and Technology Corporation at NASA Ames Research Center), who performed Cart3D the simulations and deformed the surface triangulations - in addition to providing valuable data and insight throughout the course of this work.

\section{References}

\footnotetext{
${ }^{1}$ Rodriguez, D. L., Aftosmis, M. J., Nemec, M., and Smith, S. C., "Static Aeroelastic Analysis with an Inviscid Cartesian Method," 55th AIAA/ASME/ASCE/AHS/SC Structures, Structural Dynamics, and Materials Conference, 2014, pp. 1-18.

${ }^{2}$ Nguyen, D., Nguyen, N., and Trinh, K., "Finite Element Modeling and Vibration Analysis of Aeroelastic Wing Structures," 51st AIAA Aerospace Sciences Meeting including the New Horizons Forum and Aerospace Exposition, 2013, pp. 1-26.

${ }^{3}$ Nguyen, N. T., Livne, E., Precup, N., Urnes, J. M., Nelson, C., Ting, E., and Lebofsky, S., "Experimental Investigation of a Flexible Wing with a Variable Camber Continuous Trailing Edge Flap Design," 32nd AIAA Applied Aerodynamics Conference, 2014, pp. 1-37.

${ }^{4}$ W Jr. Whitlow, R. M. B., "Application of a Transonic Potential Flow Code to the Static Aeroelastic Analysis of ThreeDimensional Wings," , No. AIAA 82-0689, 1982.

${ }^{5}$ Tatum, K. E. and Giles, G., "Integrating Nonlinear Aerodynamic and Structural Analysis for a Complete Fighter Configuration," Journal of Aircraft, Vol. 25, No. 12, 1988, pp. 1150-1156.

${ }^{6}$ Aftosmis, M. J., Berger, M. J., and Adomavicius, G., "A Parallel Multilevel Method for Adaptively Refined Cartesian Grids with Embedded Boundaries," , No. AIAA 2000-0808, January 10-13 2000.

${ }^{7}$ Nichols, R. H. and Buning, P. G., "Users Manual for OVERFLOW 2.2," Technical report, NASA, 2010.

${ }^{8}$ Nguyen, T., Precup, N., Livne, E., Urnes, J., Dickey, E., Nelson, C., Chiew, J., Rodriguez, D., Ting, E., and Lebofsky, S., "Wind Tunnel Investigation of a Flexible Wing High-Lift Configuration with a Variable Camber Continuous Trailing Edge Flap Design," 33rd AIAA Applied Aerodynamics Conference at Aviation 2015 Forum, Dallas, Texas, Jun 22-26 2015.

${ }^{9}$ Chan, W., Gomez, R., Rogers, S., and Buning, P., "Best Practices in Overset Grid Generation," 32nd AIAA Fluid Dynamics Conference and Exhibit, St. Louis, Missouri, Jun 24-26 2002, AIAA-2002-3191.

${ }^{10}$ Aupoix, B. and Spalart, P. R., "Extensions of the Spalart-Allmaras Turbulence Model to Account for Wall Roughness," International Journal of Heat and Fluid Flow, Vol. 24, 2003, pp. 454-462.
} 